\title{
Experimental Evidence for Universality of Acoustic Emission Avalanche Distributions during Structural Transitions
}

\author{
Lluís Carrillo, Lluís Mañosa, Jordi Ortín, Antoni Planes, and Eduard Vives \\ Departament d'Estructura i Constituents de la Matèria, Facultat de Física, Universitat de Barcelona, \\ Diagonal 647, E-08028 Barcelona, Catalonia, Spain
}

(Received 18 May 1998)

\begin{abstract}
Acoustic emission avalanche distributions are studied in different alloy systems that exhibit a phase transition from a bcc to a close-packed structure. After a small number of thermal cycles through the transition, the distributions become critically stable (exhibit power-law behavior) and can be characterized by an exponent $\alpha$. The values of $\alpha$ can be classified into universality classes, which depend exclusively on the symmetry of the resulting close-packed structure. [S0031-9007(98)06968-3]
\end{abstract}

PACS numbers: 64.60.Ht, 05.70.Ln, 81.30.Kf

The emission of acoustic waves in the range from $\mathrm{kHz}$ to $\mathrm{MHz}$ has been acknowledged to be a characteristic of a number of diffusionless first-order structural transitions usually called martensitic. The origin of this acoustic emission (AE) is the sudden change in the local strain field resulting from the phase transition, driven by an external applied field (stress, temperature). Actually, this AE displays many similarities with the seismic waves generated during an earthquake. The amplitude and duration of the acoustic signals generated during martensitic transitions (MT) display power-law distributions, related to the lack of characteristic temporal and spatial scales in the underlying physical mechanism [1,2]. In this respect, the martensitic transition is an example of fluctuationless first-order (FLFO) transition in a disordered system [3]. FLFO phase transitions can be modeled by Ising-type Hamiltonians at $T=0$ with quenched randomness (fields, bonds, vacancies, etc.). For these models the transition takes place only when the system is driven by an external field. The evolution proceeds by avalanches joining a series of metastable states, until the system is fully transformed. When the external field is reversed, the system returns to the original structure exhibiting history dependence and hysteresis. Because of the absence of thermal fluctuations, the sequence of avalanches determining the hysteresis loop is independent of the driving rate and is reproducible from cycle to cycle if the disorder is quenched. For a particular amount of disorder a critical point has been found [4,5]: At this point the probability distributions of sizes and durations of avalanches are power law and can be characterized by critical exponents. It has been found that these exponents, corresponding to different lattice models, have very similar values, depending only on the system dimensionality [4,5]. This suggests the existence of universality classes. The goal of this Letter is to search for experimental evidence of such universality by studying the amplitude distribution of the AE detected in different systems displaying MT.

Besides MT, many first-order transitions display FLFO characteristics. Examples are ferromagnetic materials at low temperature under an applied magnetic field [6,7], precipitation of $\mathrm{H}$ in $\mathrm{Nb}$ [8], adsorption of liquid $\mathrm{He}$ on porous media [9], and superconductivity in granular $\mathrm{Al}$ films [10]. In most of these systems critical exponents of the avalanche distribution have been measured. The comparison between the values reported is difficult because the characterization of the avalanches is done with different physical magnitudes (magnetization jumps, domain size, AE amplitudes associated with crack propagation, capacity changes, resistivity, etc.). It seems clear, therefore, that a suitable test of universality needs a systematic measurement of the same physical magnitude under the same experimental conditions.

In this Letter we present systematic results for $\mathrm{Cu}$-based alloys transforming from a high temperature bcc phase [11] to a close-packed structure. These materials are HumeRothery alloys whose phase stability is controlled by the average number of conduction electrons per atom $e / a$ [12]. For these systems, it is known that the close-packed structure depends on $e / a$ : For low values of $e / a$, a monoclinic $18 R$ (Ramsdell notation) phase is obtained while, for high values, the structure is hexagonal $2 H$. The $e / a$ value that separates the two different structures corresponds to the eutectoid point $(e / a)_{\mathrm{eu}}$ in the equilibrium phase diagram, that is the point at which the bcc stability region reaches the lowest temperature. In Table I we give the composition, $e / a,(e / a)_{\mathrm{eu}}$, and the transition temperatures of the samples studied. All samples form a polydomain structure at low temperature and are single crystal in the bcc phase, except for the samples CAM1 and CAM2 which are polycrystalline in the bcc phase (grain size $\sim 100 \mu \mathrm{m}$ ). Note that sample CAN, with an $e / a$ value very close to the eutectoid point, displays (for kinetic reasons) a double stage transformation with the formation of two closepacked structures at diferent temperatures: When cooling, a fraction of the sample transforms from bcc to $2 \mathrm{H}$ and, at a lower temperature, the remaining bcc phase transforms to $18 R$ [13]. It is worth mentioning that the transition temperatures do not exhibit any systematics with the values of $e / a$. In these MT systems, transition temperatures are 
TABLE I. Properties of the samples studied in the present work. $T_{\text {cool }}$ and $T_{\text {heat }}$ correspond, respectively, to the temperatures with maximum $\mathrm{AE}$ activity for cooling and heating ramps.

\begin{tabular}{ccccccc}
\hline \hline Sample & Composition & $(e / a)$ & $(e / a)_{\text {eu }}$ & $T_{\text {cool }}\left({ }^{\circ} \mathrm{C}\right)$ & $T_{\text {heat }}\left({ }^{\circ} \mathrm{C}\right)$ & Symbol \\
\hline CAZ1 & $\mathrm{Cu}_{69.3} \mathrm{Al}_{17.0} \mathrm{Zn}_{13.7}$ & 1.44 & 1.48 & 24.1 & 26.8 & $\bigcirc \circ \otimes$ \\
$\mathrm{CAZ2}$ & $\mathrm{Cu}_{69.7} \mathrm{Al}_{23.12} \mathrm{Zn}_{7.18}$ & 1.53 & 1.48 & -13.5 & -1.8 & $\triangleleft$ \\
$\mathrm{CAZ3}$ & $\mathrm{Cu}_{67.9} \mathrm{Al}_{16.0} \mathrm{Zn}_{16.1}$ & 1.48 & 1.48 & -40.5 & -37.5 & $\nabla$ \\
$\mathrm{CAM} 1$ & $\mathrm{Cu}_{70.09} \mathrm{Al}_{24.94} \mathrm{Mn}_{4.97}$ & 1.50 & 1.46 & -8.5 & 18.6 & $\triangleright$ \\
$\mathrm{CAM} 2$ & $\mathrm{Cu}_{70.9} \mathrm{Al}_{20.0} \mathrm{Mn}_{9.1}$ & 1.40 & 1.46 & 14.6 & 29.9 & $\square$ \\
$\mathrm{CAN}$ & $\mathrm{Cu}_{68.9} \mathrm{Al}_{27.3} \mathrm{Ni}_{3.8}$ & 1.55 & 1.53 & 12.0 & 42.3 & $\triangle$ \\
& & & & 8.9 & 14.1 & $\diamond$ \\
$\mathrm{CAB}$ & $\mathrm{Cu}_{79.97} \mathrm{Al}_{23.06} \mathrm{Be}_{2.97}$ & 1.49 & 1.49 & -54.8 & -42.3 & $*$ \\
\hline \hline
\end{tabular}

known to be sensitive to defects (disorder), due to the heterogeneous character of nucleation. This disorder includes vacancies, concentration fluctuations, line and surface defects, etc., which are modified from cycle to cycle. Moreover, metastability is not only determined by these defects but also by the existence of long-range forces of elastic nature between domains.

In order to obtain comparable results for the different samples, they have been heat treated under identical conditions: 15 min annealing at $\sim 1000 \mathrm{~K}$ and cooling in air to room temperature. With this treatment a minimum defect concentration is retained [14]. Starting with these initial conditions, we have recorded the AE during the MT by performing repetitive cooling and heating ramps at $\sim 1 \mathrm{~K} / \min$ [15]. We have used a piezoelectric transducer working in a band centered at $1 \mathrm{MHz}$. Signals are amplified and stored using a digitizing oscilloscope which is capable of recording $10^{4} \mathrm{AE}$ pulses (512 points per signal at $0.5 \mathrm{MHz}$ ) during the MT. From the recorded data, we can obtain the shape of the hysteresis loop and measure the amplitude $A$ and duration $T$ of the signals. In order to perform a statistical analysis it is very important to take into account the experimental window that limits the amplitude and duration of the recorded pulses [2]. For our data the limits are $A_{\min }=16 \mathrm{mV}<A<A_{\max }=$ $700 \mathrm{mV}$ and $T_{\min }=10 \mu \mathrm{s}<T<T_{\max }=1 \mathrm{~ms}$.

In this Letter we focus on the study of the amplitude distribution $p(A)$. The distribution $p(T)$ has also been studied but the results are much more affected by the experimental window cutoffs [2]. The experimental data have been analyzed assuming a generic normalized probability distribution: $p(A) \sim \exp (-\lambda A) A^{-\alpha}$. The exponent $\alpha$ and the parameter $\lambda$ have been estimated using a maximum likelihood method [16] with a simplex minimization algorithm [17]. The interest of this fitting procedure is that it does not involve the computation of histograms which normally depend on the binning choice. This fact, together with the large number of recorded signals, is crucial for studying cycling effects and for comparing the results from sample to sample. With this method, absolute uncertainties in the exponent are about $\pm 0.3(\sim 10 \%)$. Nevertheless, if the sample is not removed from the sample holder, the error in the comparison of $\alpha$ between runs is \pm 0.1 . This good accuracy allows, for the first time, one to study the evolution of $\alpha$ with cycling.

Figure 1 shows, for sample CAZ1, the evolution with the cycling of $\alpha$ and $\lambda$ corresponding to the cooling ramps. The two series of data correspond to two heat treatments: air cooling $(\bigcirc)$ and enhanced cooling in thermal contact with a metal block at $\sim 380 \mathrm{~K}(\circ)$. The rightmost point $(\otimes)$ corresponds to a second piece of the same crystal CAZ1 which has been cycled for about 1000 cycles. The evolution shows the same qualitative features for the two heat treatments: After the $\sim 15$ initial cycles, stable values

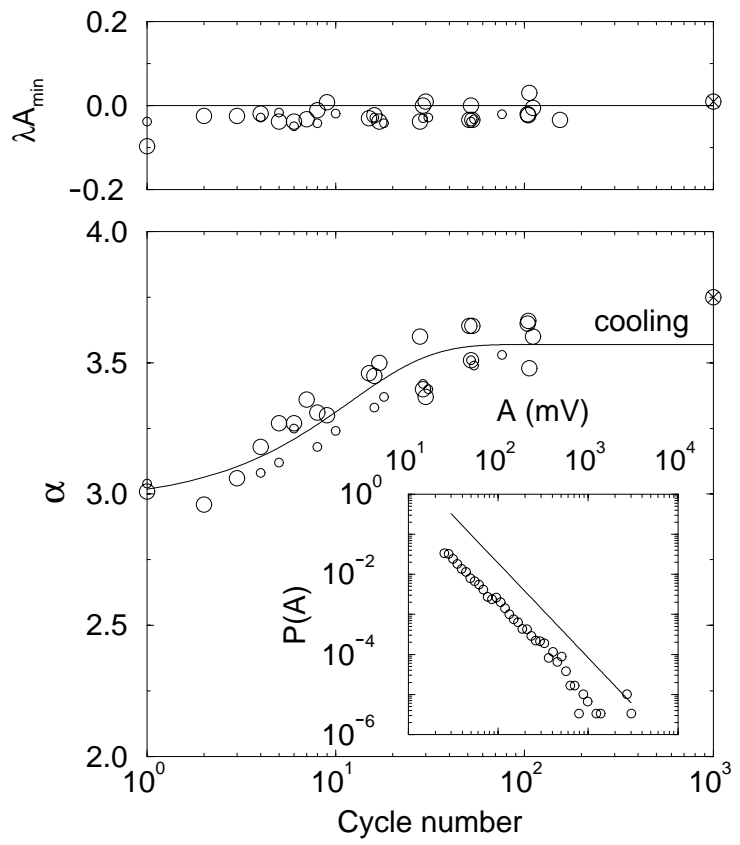

FIG. 1. Evolution of the exponent $\alpha$ and the coefficient $\lambda$ with cycling for the CAZ1 sample. Results correspond to cooling runs after two different heat treatments $(\circ, \bigcirc)$. The point $\otimes$ corresponds to a different piece of the same crystal cycled $\sim 1000$ times. The line is a guide to the eyes. The inset shows an example of the avalanche distribution obtained by overlapping data from cooling runs 16 and 17, recorded with amplification gains 53 and $44 \mathrm{~dB}$, respectively. The solid line corresponds to a power-law distribution with exponent $\alpha=3.4$ (note that we have used logarithmic bins for the histogram and, therefore, the slope is $\alpha-1=2.4$ ). 
of $\alpha$ and $\lambda$ are obtained. At this stage the values are constant with cycling, within errors, and coincide for both heat treatments. The solid line corresponds to an exponential evolution towards a constant value with a characteristic number of cycles $N_{c}=12$. Note that the values of $\lambda$ are low compared to $1 / A_{\min }$ and only a small, if any, change in the initial cooling ramp is observed. The inset corresponds to the $p(A)$ histogram, showing a powerlaw dependence extending over two decades.

Results in Fig. 1 show that $p(A)$ (and the corresponding hysteresis loop) tends, with cycling, to a final distribution with stable critical properties. This may be associated with a creation or redistribution of defects during cycling. Actually, for $\mathrm{Cu}-\mathrm{Zn}-\mathrm{Al}$, thermal cycling through the MT results in the creation of dislocations with well characterized Burger vectors, as has been observed by TEM [18]. The fact that $\lambda$ is already quite small from the first cycle could be associated with the large critical region found by Sethna and co-workers [4] in the random field Ising model. The effect of cycling on the avalanche distributions of heat pulses released in the stress-induced MT of a $\mathrm{Cu}-\mathrm{Zn}-\mathrm{Al}$ single crystal was studied recently [19]. Fits of the corresponding amplitude distributions, using the same algorithm as in the present research, give results indicative of a progressive elimination with cycling of thermal events with large amplitude. Although the comparison is not straightforward, the results are consistent with the increase of $\alpha$ with cycling shown in Fig. 1.

For all samples in Table I the same experimental procedure has been followed. Results corresponding to cooling and heating ramps have been treated separately. The reason for this is that we observe small diferences in the values of $\alpha$ corresponding to the forward and reverse transitions. The deviations, nevertheless, are not systematic. We cannot provide a full explanation for this behavior but several uncontrolled factors have to be taken into account: First, the total number of recorded signals is, in general, different from sample to sample and for cooling and heating runs, which affects the statistical errors in the exponents differently. Second, ultrasonic attenuation may also affect, slightly and differently, the cooling and heating runs.

Figure 2 shows the amplitude distribution, after $\sim 15$ cycles, for all samples studied. Data correspond to cooling ramps. Similar results for heating ramps are obtained. Power laws extend over more than one decade [20]. We have classified the data into two groups which clearly exhibit a different exponent. For the first group (above) we obtain $\alpha \simeq 3.1$, while for the second group (below) we obtain $\alpha \simeq 2.4$. Remarkably, the first group corresponds to samples transforming to the $18 R$ structure, while samples in the second group transform to $2 H$, except for one case (CAB) that will be discussed later. This result suggests that $\alpha$ could exhibit some universal character.

To make this result more evident we have plotted (Fig. 3) the values of the exponents for the different

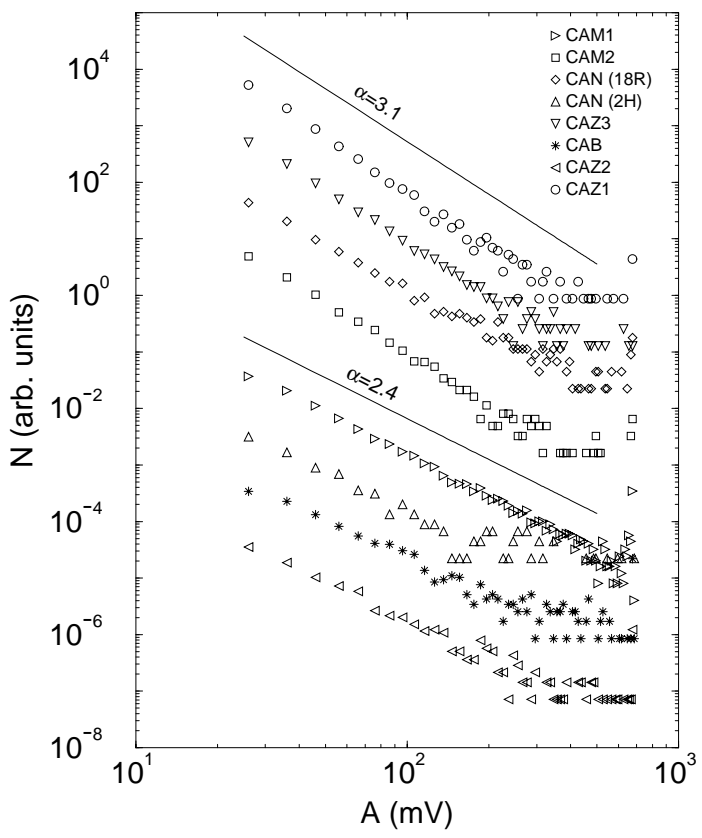

FIG. 2. Avalanche distributions obtained on cooling. The normalized histograms are shifted in order to clarify the picture. Lines are power laws with the exponents indicated above.

samples studied as a function of the reduced electronic concentration per atom $x=e / a-(e / a)_{\mathrm{eu}}$. We have represented the exponents corresponding to heating ramps (dotted symbols), cooling ramps (open symbols), and their

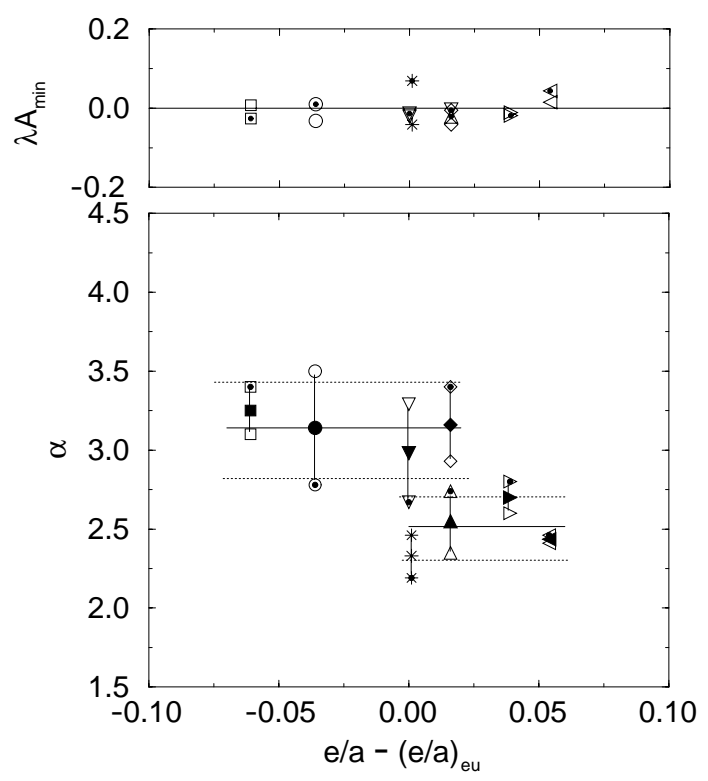

FIG. 3. Exponents $\alpha$ and coefficients $\lambda$ corresponding to the different samples studied, plotted as a function of $x=(e / a)-$ $(e / a)_{\mathrm{eu}}$. We have used the symbol shapes indicated in Table I. Open symbols correspond to cooling runs, dotted symbols to heating runs, and solid symbols to the average value. Solid lines correspond to the average values of the exponents and dashed lines show the standard deviation. 
average (solid symbols). Their difference sets up an estimation of the absolute error bars of the measurements. Data clearly show a steplike behavior: For $x<0$ (martensite with $18 R$ structure) we find $\alpha=3.1 \pm 0.2$, and for $x>0$ (martensite with $2 H$ structure) we find $\alpha=2.4 \pm$ 0.2 . This finding reinforces the hypothesis of a universal character for the exponent $\alpha$ that would only depend on the symmetry of the low temperature phase. Universality for $\alpha$ has also been found in models for FLFO phase transitions, but stronger [5]: Exponents, for the models studied, depend only on system dimensionality.

It is interesting to mention the case of $\mathrm{Cu}-\mathrm{Al}-\mathrm{Ni}$ (CAN). For this sample we have analyzed separately the data corresponding to each transition stage which correspond to a different final structure. The result found reinforces the hypothesis that the value of the exponent is related solely to the symmetry of the closepacked phase. Note also that sample $\mathrm{CAB}$ with $x \simeq$ 0 exhibits a low exponent corresponding to a sample transforming to a $2 H$ structure. Although there is some evidence that this sample transforms to a $18 R$ phase [21], it exhibits an $\mathrm{AE}$ spectrum with features similar to those of samples transforming to $2 H$. This pathology may indicate that, for this sample, $\mathrm{AE}$ (which is an out-of-equilibrium phenomena) exhibits a $2 H$ character although the final structure is $18 R$. Before concluding, we want to mention that for this class of $\mathrm{Cu}$-based alloys the entropy change at the martensitic transformation $\Delta S$ exhibits a similar steplike dependence with $x$ : For samples transforming to $18 R, \Delta S \simeq 1.3 \mathrm{~J} / \mathrm{K}$ mol while, for samples transforming to $2 H, \Delta S \simeq 1.6 \mathrm{~J} / \mathrm{K}$ mol [22]. In Cu-based alloys, $\Delta S$ is known to have its origin in the change of the vibrational spectra between the bcc and the low temperature phase. While the behavior of $\Delta S$ with $x$ refers to equilibrium properties of the MT, our results for the exponent $\alpha$ show that the same trends are operative at a dynamical level.

We can conclude that, for $\mathrm{Cu}$-based shape memory alloys, the AE amplitude distribution exponent, after enough cycling, shows a universal character independent of the chemical composition, concentration, transition temperature, heat treatment, and whether or not the system is single crystal or polycrystalline. It depends only on the symmetry of the low temperature phase. Whether the results presented in this paper can be generalized to other experimental systems transforming martensitically is still an open question, which will be interesting to analyze further.

We acknowledge E. Obradó for characterizing some of the samples. This work has received finantial support from CICyT (Project No. MAT95-0504). Ll. C. is supported by the FPI program of DGES (MEC, Spain).

[1] E. Vives, J. Ortín, Ll. Mañosa, I. Ràfols, R. PérezMagrané, and A. Planes, Phys. Rev. Lett. 72, 1694 (1994).

[2] E. Vives, I. Ràfols, Ll. Mañosa, J. Ortín, and A. Planes, Phys. Rev. B 52, 12644 (1995); I. Ràfols and E. Vives, Phys. Rev. B 52, 12651 (1995).

[3] D. A. Vul and B. N. Harmon, Phys. Rev. B 48, 6880 (1993).

[4] J.P. Sethna, K. Dahmen, S. Kartha, J. A. Krumhansl, B. W. Roberts, and J. D. Shore, Phys. Rev. Lett. 70, 3347 (1993); K. Dahmen and J. P. Sethna, Phys. Rev. Lett. 71, 3222 (1993); K. Dahmen, S. Kartha, J. A. Krumhansl, O. Perković, B. W. Roberts, and J. D. Shore, Phys. Rev. Lett. 72, 947 (1994).

[5] E. Vives, J. Goicoechea, J. Ortín, and A. Planes, Phys. Rev. E 52, R5 (1995).

[6] K. L. Babcock and R. M. Westervelt, Phys. Rev. Lett. 64, 2168 (1990).

[7] P. J. Cote and L. V. Meisel, Phys. Rev. Lett. 67, 1334 (1991).

[8] G. Cannelli, R. Cantelli, and F. Cordero, Phys. Rev. Lett. 70, 3923 (1993).

[9] M. P. Lilly, P. T. Finley, and R. B. Hallock, Phys. Rev. Lett. 71, 4186 (1993).

[10] W. Wu and P. W. Adams, Phys. Rev. Lett. 74, 610 (1995).

[11] In Cu-based alloys, the bcc phase is only stable at relatively high temperature but it can be retained at room temperature by suitable cooling. These are the conditions at which the MT takes place.

[12] T. B. Massalski and U. Mizutani, Prog. Mater. Sci. 22, 151 (1978).

[13] C. M. Friend et al., Scr. Metall. Mater. 24, 1641 (1990).

[14] M. Ahlers, Prog. Mater. Sci. 30, 135 (1986).

[15] The results show no dependence with the cooling and heating rate in the range 0.1 to $10 \mathrm{~K} / \mathrm{min}$.

[16] R. Barlow, Statistics (Wiley, New York, 1989).

[17] W. H. Press, B.P. Flannery, S. A. Teukolsky, and W. T. Vetterling, Numerical Recipes (Cambridge University Press, New York, 1990).

[18] D. Ríos-Jara and G. Guénin, Acta Metall. 35, 109 (1987); J. Pons, F. C. Lovey, and E. Cesari, Acta Metall. Mater. 38, 2733 (1991).

[19] Ll. Carrillo and J. Ortín, Phys. Rev. B 56, 11508 (1997).

[20] This range, as shown in Fig. 1, may be increased, to more than two decades, by mixing data recorded with different amplifier gains. We have checked that this procedure does not render significantly different $\alpha$ exponents.

[21] S. Belkhala, Ph.D. thesis, Institut National de Sciences Appliqueés (90 ISAL 0093), Lyon, France, 1990.

[22] E. Obradó, Ll. Mañosa, and A. Planes, Phys. Rev. B 56, 20 (1997). 